\title{
Sciendo
}

RESEARCH PAPERS FACULTY OF MATERIALS

SCIENCE AND TECHNOLOGY IN TRNAVA

SLOVAK UNIVERSITY OF TECHNOLOGY

IN BRATISLAVA

2021, Volume 29, Number 49

DOI 10.2478/rput-2021-0024

\section{CASE STUDY OF BUILDING A DIGITAL TWIN FOR A PRODUCTION SYSTEM}

\author{
Fedor BURČIAR ${ }^{1}$, Pavel VAŽAN ${ }^{1}$, Simona PULIŠOVÁ ${ }^{1}$ \\ ${ }^{1}$ Slovak UNIVERSity OF TECHNOLOGY IN BRATISLAVA, \\ FACULTY OF MATERIALS SCIENCE AND TECHNOLOGY IN TRNAVA, \\ INSTITUTE OF APPLIED INFORMATICS, AUTOMATION AND MECHATRONICS, \\ Ulica JÁnA BotTu 2781/25, 91724 TRNAVA, SlovaK REPUBliC \\ e-mail: fedor.burciar@stuba.sk,pavel.vazan@stuba.sk,xpulisova@stuba.sk \\ Received 27 August 2021, Accepted 25 October 2021, Published 24 November 2021
}

\begin{abstract}
As the term of Industry 4.0 becomes more and more relevant with each passing day, it is up to researchers and companies to find solutions to integrating all the technologies it covers. One of those technologies, even though not highly developed, is simulation and building CyberPhysical Systems for gathering data and improving the production processes. In the research described in this paper, we focused on integrating production data with simulation models in order to make the process of understanding and learning about complex production systems as simple and as quick as possible. This paper contains three sections. The first one introduces the theoretical fundamentals of our research. The second one focuses on the methods used to create a digital model of production system. The final one discusses the results of the conducted experiments, and their impact on further research.
\end{abstract}

\section{Keywords}

Digital Twin, Simulation, Witness, AFB Factory

\section{INTRODUCTION}

Since the introduction of a term of "Industry 4.0 (I4.0)" proposed as a name for the latest industrial revolution in 2011, multiple authors have made attempts at defining their own interpretation of this expression. Majority of those authors agreed it consisted of the key topics related to Cyber-physical systems (CPS), Internet of Things (IoT), Big Data, Simulation and others [10]. I4.0 can be viewed as a means of digitization and automation of not only production processes, but an entire company as a whole [1].

Being one of the core concepts of I4.0, simulation, and more notably Digital Twins (DT), offers companies a way to optimize their processes, ranging from product life-cycle management (PLM) through maintenance to logistics [11]. Digital twins, however, rely on large amounts of data if they are supposed to represent a reliable digital version of a real-life entity, 
whether it is a production or transport system. This presents a big problem owing to the lack of data processing solutions that would effectively handle real-time multi-source information [9]. The existing solutions are often followed by inconsistent data format, missing data or logic errors [2]. Many people may fall into a misconception that digital models used in simulation are indeed a digital twin [7]. Authors defining DT (Hughes 2018; Negri et al. 2017; Tao et al. 2017; Garetti et al. 2012; Siemens 2018 and others) imply that DT is a virtual duplication of a physical entity, but a digital model does not necessarily have to hold the latest information about its physical counterpart, since the information within the digital model can be generated and manipulated. Therefore, DT can be considered a digital model, but a digital model cannot always be considered a digital twin [3]. Kritzinger et al. (2018) proposed the classification of digital models based on distinctive differences between Digital Model, Digital Shadow and a Digital Twin, which are often used as synonyms in simulation. Their most notable difference lies in the level of data integration between the physical and digital counterparts. According to Kritzinger, the differences are: 1) The data between a Digital Model and its physical counterpart is executed manually in both directions, and therefore the changes in one do not affect the other; 2) Digital Shadow (DS) is automatically updated upon any changes occurring in a physical system, but data transmission from DS back to its counterpart has to be performed manually; 3) Data flow between a physical system and its Digital Twin is fully integrated, and thus performed automatically both ways [4].

\section{METHODOLOGY}

This paper presents a case study demonstrating a specific procedure of building a DT for a production system, where not all the process data is accessible to the end user. The paper also contains a sample of preliminary tests carried out on the DT with different data sets. The production system used as a test subject in the research described in this paper was Agro, Food and Beverages Factory (AFB Factory) of Festo Didactic.

\section{Data Acquisition}

Acquiring large amounts of required data for building a DT involved using the multiple data acquisition methods [12], some of which were considered sub-optimal, but had to be used until a better, more accurate method was proposed. The data required for building a DT involves product flow, types of products entering and leaving the system, number of machines in the system, duration of all the operations performed by machines, etc. In general, data can be divided into three categories, based on its accessibility and availability [5,6]: available data easily obtainable from the system and ready to be used in the modeling process; non-available but accessible through certain methods of data acquisition; neither available nor accessible by any means - estimates are generally used instead of the data. The data categorized as available and easily accessible in the production system was for example machine organization, types of products being processed and batch size [6]. The data that required some form of analysis was the storage capacity, conveyor capacity, and storage methods for finished and unfinished products. The final category of data which required some form of estimation was the speed of conveyor belt within the system and duration of manipulation cycles with individual products. Both of these examples of data could be gathered from other parts of the named production system, but required usage of more advanced methods and more time.

\section{Modelling}

Before attempting to create a digital model for the above-mentioned production system, it was necessary to develop a conceptual model (Fig. 2) which would serve as a guideline for creating the digital model. The main purpose of the conceptual model was to illustrate the exact 
order in which the products are processed within the production system. This information would later be used to correctly define the input/output rules within the digital model itself [8]. Having gathered all the required data from the MES and process observation, we proceeded to creating a functioning digital model of a production system. For this purpose, the software available at the Department of Applied Informatics, Automation and Mechatronics was used, namely WITNESS Horizon by Lanner Group. Since the subject of the study was a production system operating with both discrete events (bottle transport and handling) and fluid materials (bottle filling), it was mandatory that the proposed model was fully functional in its discrete-event state first. While building the digital model, it was necessary to substitute certain stations with abstractions equal in functionality to their physical counterpart, but offering an option of a simplified definition and easier manipulation on an occasion when changes needed to be made [8]. The digital model (Fig. 1) was visually separated into three regions, based on the functionality of machines in that region: transport and logistics, automated production, filling and packaging, while those regions were interconnected by a conveyor belts transporting the products (bottles). Each of the aforementioned stations had its cycle and work times specified and adjusted according to the manufacturing data gathered [7]. This step was necessary in order to make the digital model represent as close of a copy of the physical system as possible.
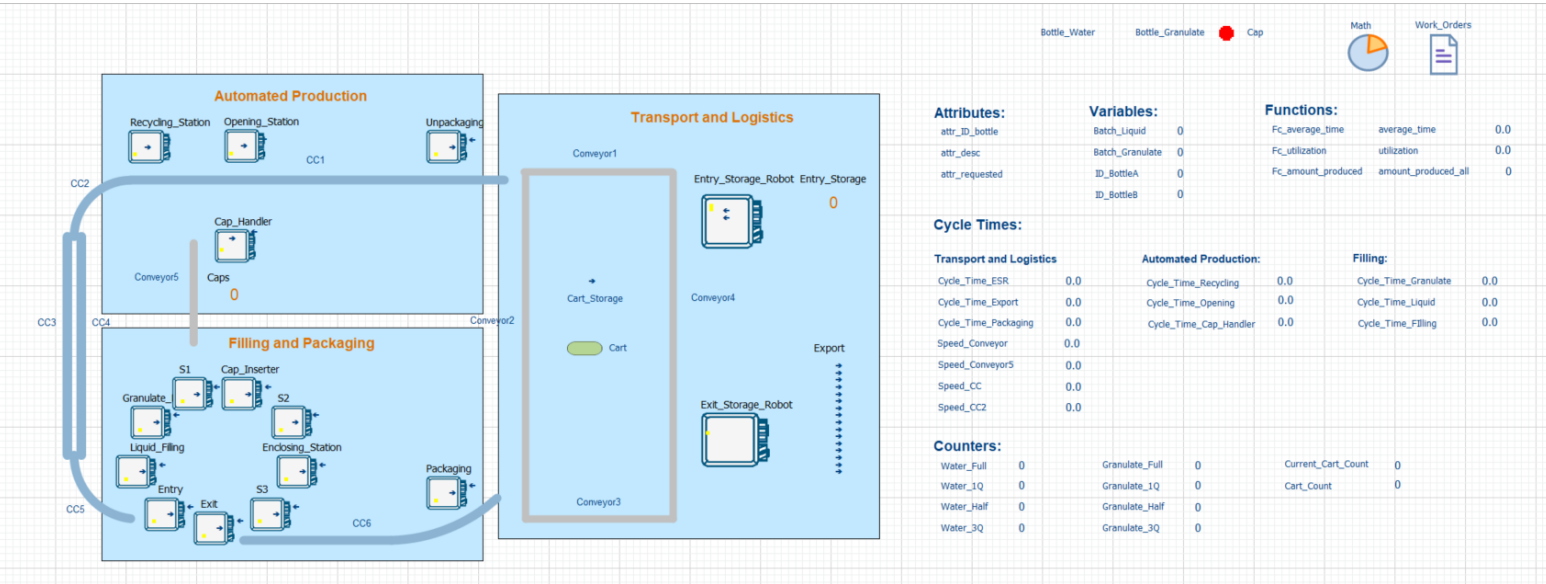

Fig. 1 2D Digital Model of AFB Festo

The station responsible for filling and enclosing the bottles in a physical system operates in nine different positions, through which all the bottles, regardless of their type, have to pass. Not all bottles are processed at each of those positions, but rather based on their type, each position has a different operation queued for the bottle of that type. The station was separated into a series of nine different machines within the digital model, in order to replicate the process with the highest precision, and an option to modify the parameters of each position individually. The model handles three types of items: water bottles, granulate bottles and bottle caps, with each of them subject to different actions performed on them during the production process.

\section{Simulation}

Having built the model, we ran validation tests in the form of simulation with different data sets, which was the next step towards finalizing the model. Every Digital Model that is to be converted into a Digital Twin should be capable of handling the same types of requests as its physical counterpart. In our particular case, the entry data we used was multiple sets of work orders requiring different amounts of bottles of each type filled by varying amounts of the corresponding contents. The main goal of these experiments was to assure that there were no 
bottlenecks within the model, and that digital model handled the work orders correctly. In order to monitor the important parts of the simulation process, we proposed simple mathematical formulas that would keep track of the key value indicators within the model, and help evaluate overall performance of the ongoing simulation. The first key indicator (1) was the formula tracking the number of finished products of each subtype (based on the volume of contents in the bottles) and the sum of all finished products. It was also an indicator of the highest priority in the Digital Model validation, since the amount of finished products had to be equal to the amount of the products requested by MES.

$$
\text { Total }=\sum_{i=1}^{4} \sum \text { WaterBottle }_{i}+\sum_{j=1}^{4} \sum \text { GranulateBottle }_{j}
$$

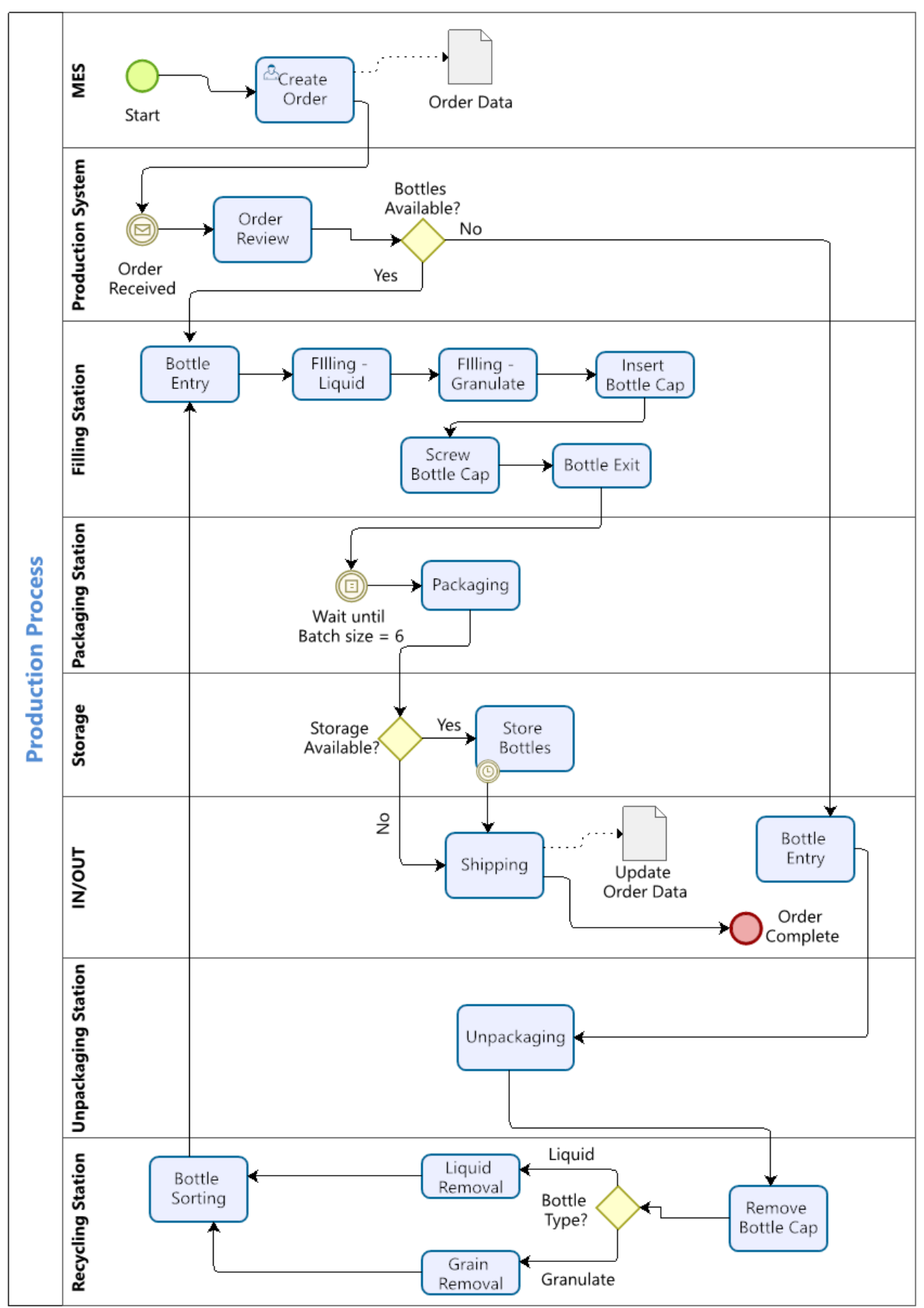


Fig. 2 Conceptual model

Tracking the utilization of each individual work station or machine within the model was another priority. We used a software function (PUtil) to track each individual machine $(X)$ in its busy state, and then proposed a formula to get a rough estimate of the system utilization within the Digital Model, while also keeping track of utilization of each individual machine.

$$
\text { Utilization }=\frac{\sum_{i=1}^{16} \operatorname{PUtil}\left(X_{\mathrm{i}}\right)}{16} .
$$

In order to gain all the fundamental information from the validation process, we proposed another formula within the model, the one that would keep track of average time spent in the model for each type of product from start to finish, and of all bottles together.

$$
\text { AvgTime }=\frac{\sum_{i=1}^{X} \text { AFlow }\left(\text { BottleWater }_{i}\right)+\sum_{j=1}^{Y} \text { AFlow }\left(\text { Bottle Granulate }_{j}\right)}{2} .
$$

AFlow is a function specific for the software we used; its purpose is to track the average time spent in the production process of any specified item. Variables $X$ and $Y$ in the formula are defined by the number of requested items of specific type, and can vary from one work order to another.

\section{RESULTS AND DISCUSSION}

During the testing phase of the digital model, we ran multiple experiments with varying data, with the biggest differences being the intervals of incoming work orders. The experiments were mostly focused on monitoring the utilization of all the machines and work station within the model in an attempt of finding problematic spots. Each of the experiments carried out on the model consisted of 15 work orders for six bottles, totaling in 90 bottles that needed to be processed. The model was initialized from the current state reflecting the real state of the production system, in order to simulate a system with an ongoing production. Products that were already in the system were excluded from the final results showing the number of finished products and average time spent in the process.

\section{Experiment 1}

The Experiment duration was measured from the time of receiving the first work order until the export of the last remaining bottle in the system. Duration of Experiment 1 was 2435 seconds, and intervals between work orders were specified at random between 10 and 100 seconds. During that time, by closely observing the ongoing simulation, we were able to deduce that there was a major bottleneck within the model located at the "Unpackaging Station". After revising the data, it was clear that the results were indeed skewed by the bottleneck, resulting in alarmingly high average time spent in the system, peaking at 1078 seconds. Simulation results also showed overall utilization of the system being at $36.5 \%$ across all machines in the production system. Running the same Experiment multiple times, it was found that the system reached its limit and was unable to handle the interval at which the work orders arrived into the system. The amount of time the "Cap_Handler" machine was blocked is given by a very low volume of storage for bottle caps in the production system, and is therefore considered irrelevant for those results, as it also has no impact on the overall flow of bottles in the system.

On the other hand, the amount of time the "Unpackaging" machine was blocked (53\%) had to be addressed before coming to other conclusions about the efficiency of the production 
system. In an attempt to achieve that, we generated another set of test data, which had the work orders spaced further apart at consistent 100 seconds in between.

\section{Experiment 2}

Having the work orders adjusted, Experiment 2 showed that there was a significant improvement in the "\% Blocked" department, up to $32.44 \%$, as shown in Table 1 . The improvement had also impact on the amount of time the bottles had spent in the system, reaching 690 seconds at its peak, which was a $36 \%$ increase when compared to Experiment 1. The overall utilization of the production system also went up slightly, resulting in a gain of $0.1 \%$ when compared to Experiment 1 . The Table below shows all the data gathered about machines during Experiment 2.

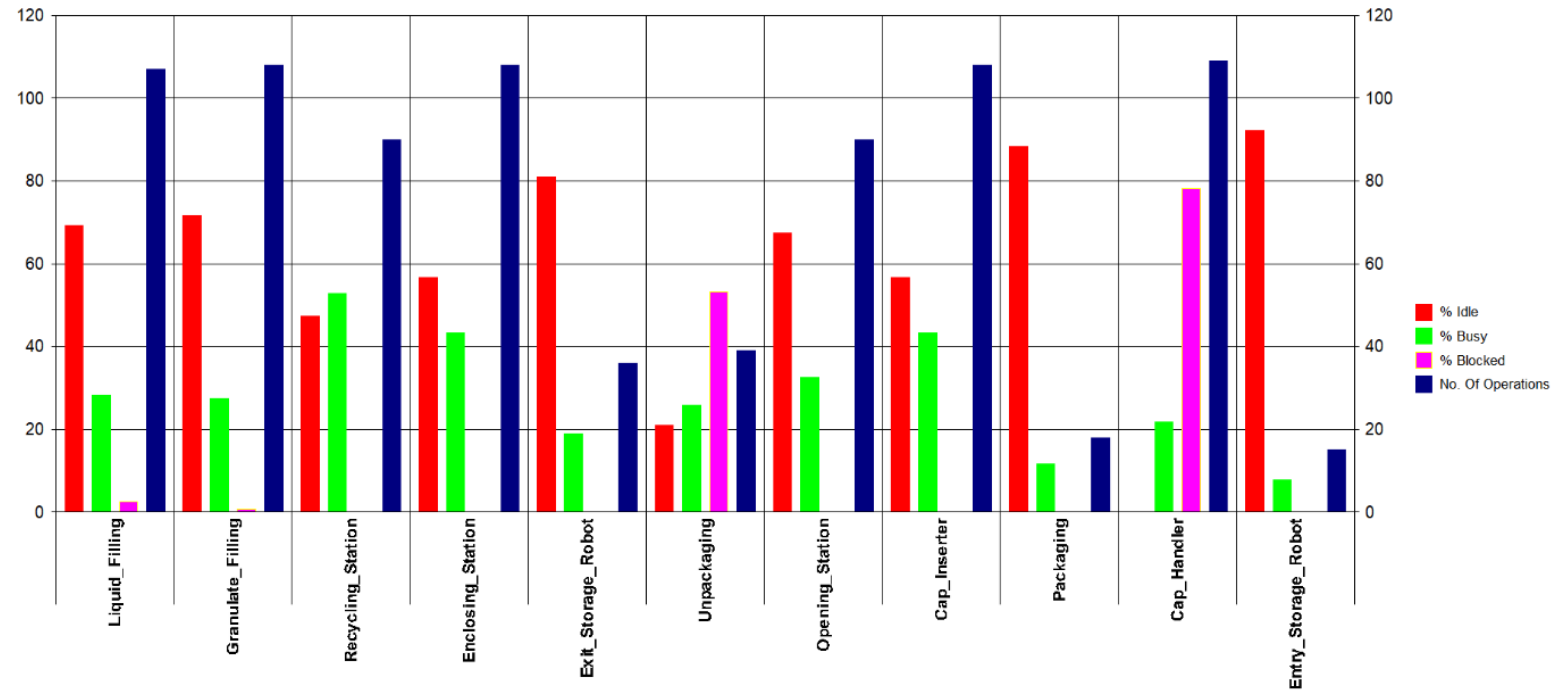

Fig. 3 Results of the experiment 1

Increasing the interval between work orders in Experiment 2 yielded much better overall results. Based on that, we proceeded with further increasing the interval to 150 seconds per work order, and ran another set of experiments with a newly generated set of data.

\begin{tabular}{|l|c|c|c|c|}
\hline \multicolumn{1}{|l|}{ Table 1 Results of Experiment \#2 } & \% Idle & \% Busy & \% Blocked & No. of Operations \\
\hline Fluid_Filling & 69.10 & 28.38 & 2.52 & 107 \\
\hline Granulate_Filling & 71.63 & 27.67 & 0.70 & 108 \\
\hline Recycling_Station & 46.99 & 53.01 & 0.00 & 90 \\
\hline Enclosing_Station & 56.43 & 43.57 & 0.00 & 108 \\
\hline Exit_Storage_Robot & 75.35 & 24.65 & 0.00 & 47 \\
\hline Unpackaging & 35.08 & 32.48 & 32.44 & 49 \\
\hline Opening_Station & 67.33 & 32.67 & 0.00 & 90 \\
\hline Cap_Inserter & 56.43 & 43.57 & 0.00 & 108 \\
\hline Packaging & 88.24 & 11.76 & 0.00 & 18 \\
\hline Cap_Handler & 0.00 & 21.98 & 78.02 & 109 \\
\hline Entry_Storage_Robot & 92.13 & 7.87 & 0.00 & 15 \\
\hline
\end{tabular}

\section{Experiment 3}


The improvements in the production process during Experiment 3 in the series were evident already during the simulation process, without looking at and analyzing the recorded data. The bottleneck at the "Unpackaging" station was close to non-existent, and the flow of bottles significantly improved. The post-simulation data analysis confirmed what was shown during the simulation, reducing the "\% Blocked" state at "Unpackaging" machine to $1.25 \%$. The average time the bottles spent in the system was also reduced to roughly 450 seconds, which could be considered a big improvement when comparing these results to the previous two experiments. The increase in the interval between work orders also had some minor negative effects on the overall utilization of the system, which ended at $34.3 \%$ after Experiment 3.

\begin{tabular}{|l|c|c|c|c|}
\hline \multicolumn{7}{|l|}{ Table 2 Results of Experiment \#3 } & \% Idle & \% Busy & \% Blocked & No. of Operations \\
\hline Name & 71.60 & 26.08 & 2.32 & 107 \\
\hline Fluid_Filling & 77.77 & 21.59 & 0.64 & 108 \\
\hline Granulate_Filling & 51.29 & 48.71 & 0.00 & 90 \\
\hline Recycling_Station & 59.97 & 40.03 & 0.00 & 108 \\
\hline Enclosing_Station & 66.75 & 33.25 & 0.00 & 69 \\
\hline Exit_Storage_Robot & 55.82 & 42.93 & 1.25 & 71 \\
\hline Unpackaging & 69.97 & 30.03 & 0.00 & 90 \\
\hline Opening_Station & 59.97 & 40.03 & 0.00 & 108 \\
\hline Cap_Inserter & 89.19 & 10.81 & 0.00 & 18 \\
\hline Packaging & 0.00 & 20.20 & 79.80 & 109 \\
\hline Cap_Handler & 92.77 & 7.23 & 0.00 & 15 \\
\hline Entry_Storage_Robot & & & & \\
\hline
\end{tabular}

\section{CONCLUSION}

Based on the performed experiments, we were able to deduce that the interval between individual work orders has the biggest impact on the performance of this production system. Further experiments had to be carried out using the created digital model in order to find the optimal interval for the arriving work orders. Alternative measures can be taken while optimizing the production system, but it would require making technological adjustments on the production system, such as adjusting cycle times for different machines, increasing storage capacities, or adding additional parallel work stations to the existing ones. Finding better methods of data acquisition and their extraction from the physical production system are also necessary for improving the main flaws of the digital model and its transition into a digital twin operating with the real time data collected from its physical counterpart. All of the abovementioned goals are subjects of future research.

\section{Acknowledgement}

This publication was written with the support of the Operational Program Integrated Infrastructure within the project of Výskum v sieti SANET a možnosti jej d'alšieho využitia a rozvoja (Research into the SANET and options of its further utilisation and development), code ITMS 313011W988, co-financed by the ERDF.

\section{References}

[1] TAY, S., TE CHUAN, L., AZIATI, A., AHMAD, A. N. A. 2018. An Overview of Industry 4.0: Definition, Components, and Government Initiatives. Journal of Advanced Research in Dynamical and Control Systems. 10(14 - Special Issue) pp. 1379-1387, ISSN 1943-023X 
[2] XIYU G. et al 2021 J. Phys.: Conf. Ser. 1983012104

[3] SHAO, G., LAROQUE, Ch., LENDERMANN, P., JAIN, S., LEE, H. L., ROSE, O. 2019. Digital Twin for Smart Manufacturing: The Simulation Aspect. 2019 Winter Simulation Conference (WSC). 2085-2098. ISSN 1558-4305.

[4] KRITZINGER, W., KARNER, M., TRAAR, G., HENJES, J., SIHN, S. 2018. Digital Twin in manufacturing: A categorical literature review and classification, IFAC-PapersOnLine, 51(11), 1016-1022. ISSN 2405-8963.

[5] Lanner Group, Ltd. 2016. Learning WITNESS Book One Manufacturing Performance Edition. ISNB 978-1-291-47674-3.

[6] Lanner Group, Ltd. 2016. Learning WITNESS Book Two Manufacturing Performance Edition. ISNB 978-1-291-47878-5.

[7] PULIŠOVÁ, S.: Digital twin of production line. [Master thesis] - Slovak University of Technology in Bratislava. Faculty of Materials Science and Technology in Trnava; Institute of Applied Informatics, Automation and Mechatronics. - Trnava: MTF STU, 2021. 89p

[8] ČERVEŇANSKÁ, Z., VAŽAN, P., JUHÁS, M., JUHÁSOVÁ, B. 2021. MultiCriteria Optimization in Operations Scheduling Applying Selected Priority Rules. Appl. Sci. 2021, 11, 2783.

[9] GRIEVES, M., VICKERS, J. 2017. Digital Twin: Mitigating Unpredictable, Undesirable Emergent Behavior in Complex Systems. In: Transdisciplinary Perspectives on Complex Systems. Springer, Cham, pp. 88-113. ISBN 978-3-319-38756-7.

[10] UHLEMANN, T. H.-J., LEHMANN, Ch. STEINHILPER, R. 2017. The Digital Twin: Realizing the Cyber-Physical Production System for Industry 4.0, Procedia CIRP 61 (2017) 335 - 340.

[11] TAY, S. I., LEE, T. C., HAMID, N. A. A., AHMAD, A. N. A., An Overview of Industry 4.0: Definition, Components, and Government Initiatives, Journal of Advanced Research in Dynamical and Control Systems, Vol. 10, 14-Special Issue, 2018.

[12] RESMAN, M.; PROTNER, J.; SIMIC, M.; HERAKOVIC, N. A Five-Step Approach to Planning Data-Driven Digital Twins for Discrete Manufacturing Systems. Appl. Sci. 2021, 11, 3639.

\section{ORCID}

Pavel Važan 0000-0001-6943-2635 